\title{
COMPUTER SIMULATION OF EFFECT OF THERMAL FLUCTUATION ON MAGNETIC RECORDING MEDIA
}

(Department of Computer Science, University of Electro-Communications)

\section{Introduction}

The effect of thermal fluctuation on longitudinal and perpendicular recording media was investigated by computer simulation. Thermal effect for the ideal recording state in three years was simulated, and the output signal at each calculation step was obtained from the magnetization pattern. The calculation result shows that the perpendicular recording is superior to the longitudinal recording with the increase of the recording density from the viewpoint of thermal fluctuation.

\section{Effect of Thermal Fluctuation on Magnetization Pattern}

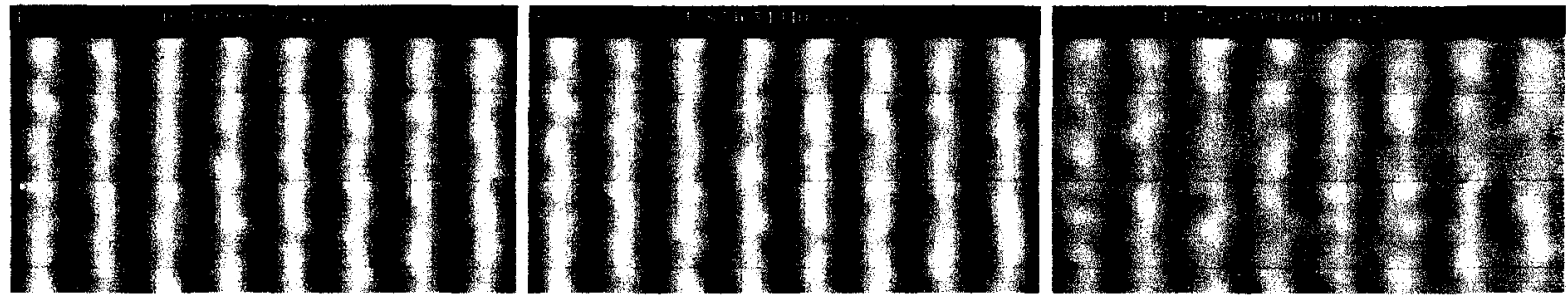

(a)L:T=0s, Signal $=100 \%$

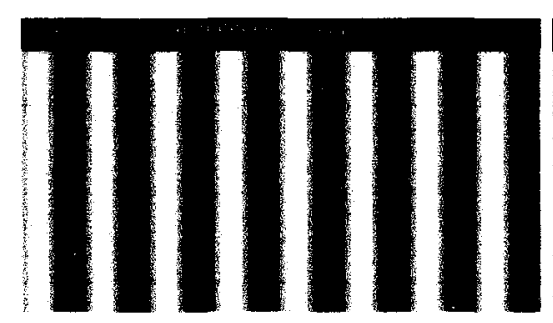

(d) $\mathrm{P}: \mathrm{T}=0 \mathrm{~s}$, Signal $=100 \%$ (b) $\mathrm{L}: \mathrm{T}=1 \mathrm{~s}$, Signal $=92 \%$

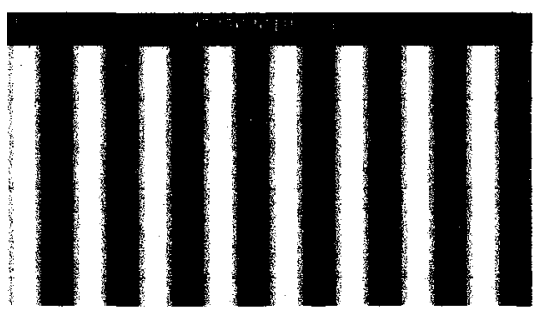

(e)P:T=1s, Signal $=98 \%$ (c) L:T $=10000$ s, Signal $=55 \%$

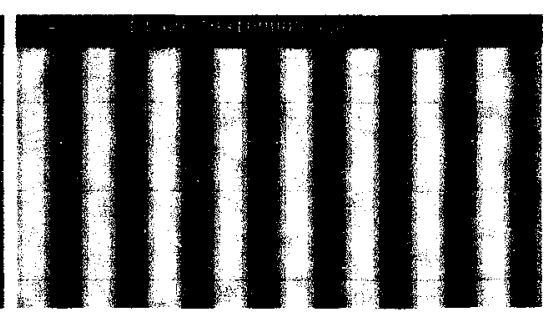

(f) $P: T=10000 s$, Signal $=63 \%$

Simulated MFM image of magnetization pattern (408KFCI, D=100A, H=150A)

\section{Effect of Thermal Fluctuation on Output Signal}

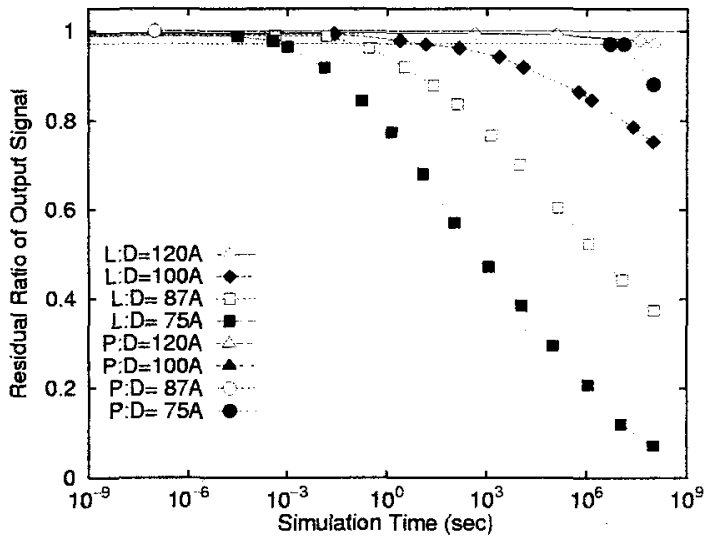

(a) Dependence of residual ratio of output signal on time $(408 \mathrm{KFCI})$.

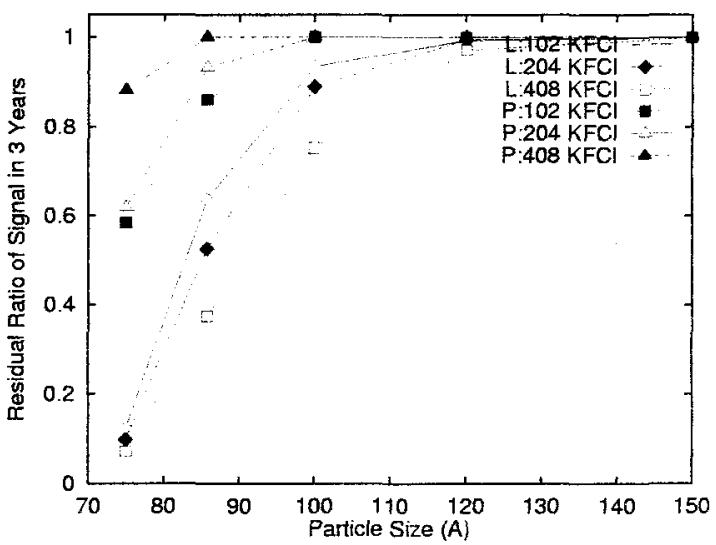

(b) Dependence of residual ratio of output signal in three years on recording density. 